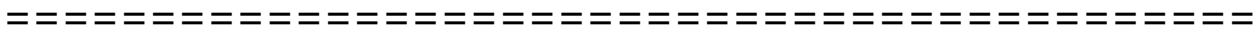

$\begin{array}{lllllllllllllllllllll}S & \mathrm{U} & \mathrm{P} & \mathrm{P} & \mathrm{L} & \mathrm{E} & \mathrm{M} & \mathrm{E} & \mathrm{N} & \mathrm{T} & \mathrm{A} & \mathrm{R} & \mathrm{Y} & \mathrm{M} & \mathrm{A} & \mathrm{T} & \mathrm{E} & \mathrm{R} & \mathrm{I} & \mathrm{A} & \mathrm{L}\end{array}$

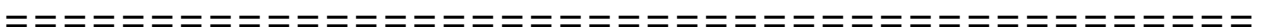

\title{
Solid-state NMR of a paramagnetic DIAD-Fe(II) catalyst: sensitivity, resolution enhancement, and structure-based assignment
}

Gwendal Kervern, Guido Pintacuda, Yong Zhang, Eric Oldfield, Charbel Roukoss, Emile Kuhn, Eberhardt Herdtweck, Jean-Marie Basset, Sylvian Cadars, Anne Lesage, Christophe Coperet and Lyndon Emsley
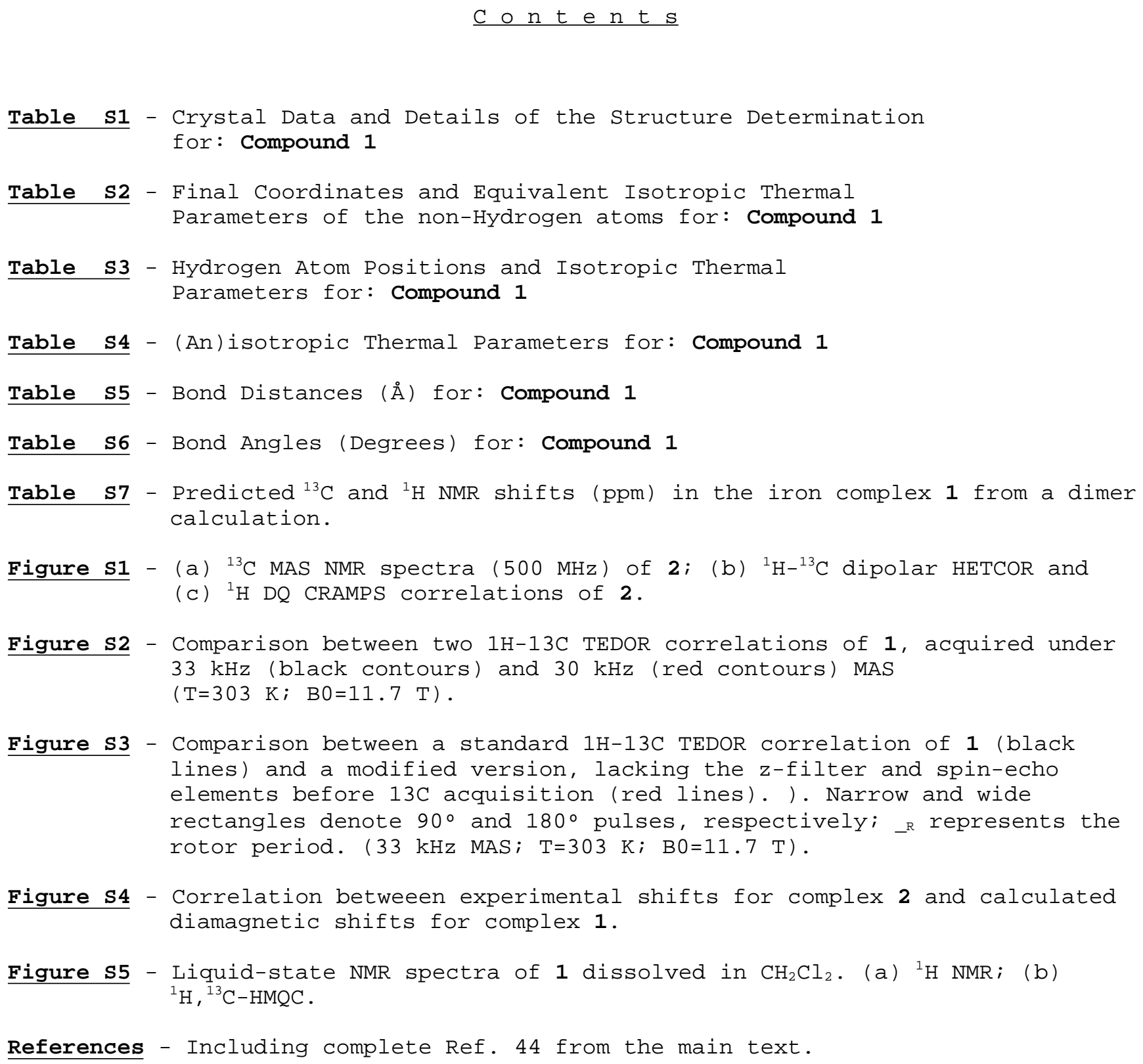
2,3-dimethyl-1,4-[(2',6')-diisopropylphenyl]-NN'-diazadiene bis-chloride iron (1) Ellipsoids at $50 \%$

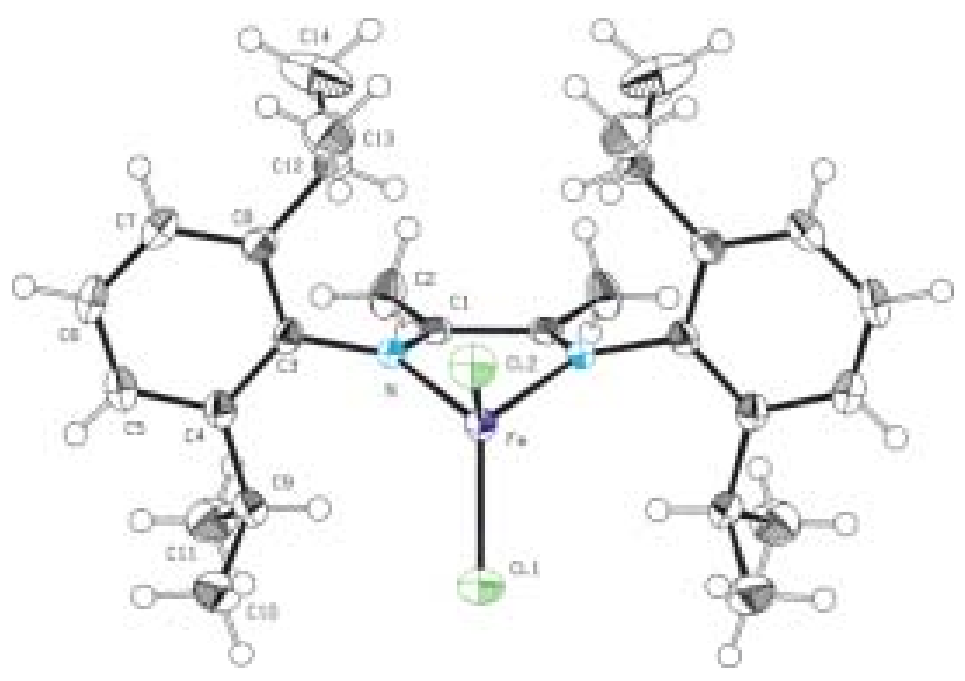


Table S1 - Crystal Data and Details of the Structure Determination for: Compound 1

Crystal Data

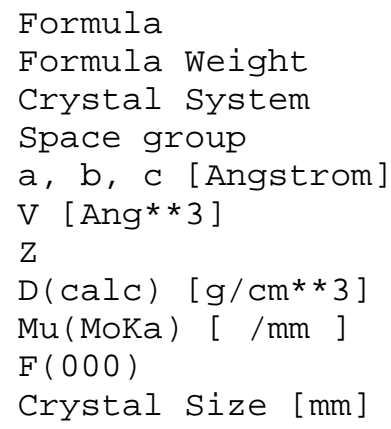

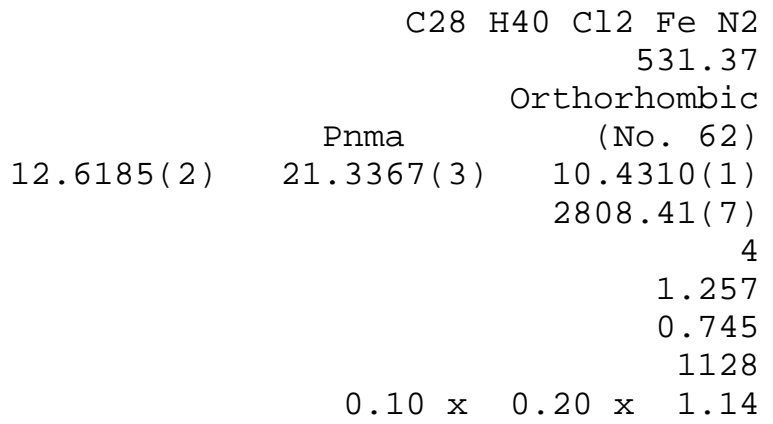

Data Collection

Temperature ( $\mathrm{K}$ )

Radiation [Angstrom]

Theta Min-Max [Deg]

Dataset

Tot., Uniq. Data, R(int)

Observed data [I $>2.0$ sigma(I) ]

Refinement

Nref, Npar

$R$, wR2, $S$

Max. and Av. Shift/Error

Min. and Max. Resd. Dens. [e/Ang^3]
173

MoKa $\quad 0.71073$

1.9, 25.3

$-15: 15 ;-25: 25 ;-12: 12$

$65010, \quad 2646,0.064$

2504

Table S2 - Final Coordinates and Equivalent Isotropic Thermal Parameters of the non-Hydrogen atoms for Compound 1

\begin{tabular}{|c|c|c|c|c|}
\hline Atom & $\mathrm{x}$ & $\mathrm{y}$ & $\mathrm{z}$ & $\mathrm{U}(\mathrm{eq}) \quad\left[\mathrm{Ang}^{\wedge} 2\right]$ \\
\hline---- & --- & --- & --- & ----------- \\
\hline $\mathrm{Fe}$ & $0.18492(3)$ & $1 / 4$ & $0.27977(3)$ & $0.0201(1)$ \\
\hline $\mathrm{Cl1}$ & $0.14552(5)$ & $1 / 4$ & $0.07156(6)$ & $0.0322(2)$ \\
\hline $\mathrm{Cl} 2$ & $0.35611(5)$ & $1 / 4$ & $0.32729(7)$ & $0.0342(2)$ \\
\hline $\mathrm{N}$ & $0.08962(11)$ & $0.18902(6)$ & $0.38814(13)$ & $0.0177(4)$ \\
\hline $\mathrm{C} 1$ & $0.00586(13)$ & $0.21462(8)$ & $0.43378(15)$ & $0.0191(5)$ \\
\hline $\mathrm{C} 2$ & $-0.08325(17)$ & $0.18063(10)$ & $0.4965(2)$ & $0.0323(6)$ \\
\hline $\mathrm{C} 3$ & $0.11004(13)$ & $0.12296(8)$ & $0.40775(17)$ & $0.0206(5)$ \\
\hline C4 & $0.07903(14)$ & $0.07919(8)$ & $0.31527(17)$ & $0.0237(5)$ \\
\hline C5 & $0.10540(16)$ & $0.01646(9)$ & $0.3379(2)$ & $0.0311(6)$ \\
\hline $\mathrm{C} 6$ & $0.15962(16)$ & $-0.00136(9)$ & $0.4465(2)$ & $0.0329(6)$ \\
\hline $\mathrm{C} 7$ & $0.18884(16)$ & $0.04269(9)$ & $0.5363(2)$ & $0.0307(6)$ \\
\hline $\mathrm{C} 8$ & $0.16526(14)$ & $0.10595(9)$ & $0.51933(18)$ & $0.0258(5)$ \\
\hline C9 & $0.01783(16)$ & $0.09729(9)$ & $0.19582(18)$ & $0.0278(6)$ \\
\hline $\mathrm{C} 10$ & $0.0769(2)$ & $0.07882(12)$ & $0.0741(2)$ & $0.0436(8)$ \\
\hline C11 & $-0.0932(2)$ & $0.06883(13)$ & $0.1979(2)$ & $0.0437(8)$ \\
\hline $\mathrm{C} 12$ & $0.19895(19)$ & $0.15340(10)$ & $0.6205(2)$ & $0.0385(7)$ \\
\hline $\mathrm{C} 13$ & $0.3184(2)$ & $0.15432(14)$ & $0.6394(3)$ & $0.0522(9)$ \\
\hline C14 & $0.1428(3)$ & $0.1403(2)$ & $0.7481(3)$ & $0.0707(13)$ \\
\hline
\end{tabular}


Table S3 - Hydrogen Atom Positions and Isotropic Thermal Parameters for Compound 1

$\begin{array}{lcccc}\text { Atom } & \mathrm{X} & \mathrm{Y} & \mathrm{z} & \mathrm{U}(\mathrm{iso}) \text { [Ang^2] } \\ ---- & --- & --- & -------- \\ \text { H21 } & -0.0729(18) & 0.1388(12) & 0.492(2) & 0.046(7) \\ \text { H22 } & -0.087(2) & 0.1931(13) & 0.584(3) & 0.070(9) \\ \text { H23 } & -0.149(2) & 0.1913(11) & 0.458(2) & 0.048(7) \\ \text { H51 } & 0.0837(17) & -0.0133(11) & 0.281(2) & 0.036(6) \\ \text { H61 } & 0.1791(17) & -0.0445(11) & 0.460(2) & 0.039(6) \\ \text { H71 } & 0.2267(16) & 0.0302(10) & 0.610(2) & 0.031(5) \\ \text { H91 } & 0.0096(15) & 0.1412(10) & 0.1968(17) & 0.022(5) \\ \text { H101 } & 0.0361(18) & 0.0934(11) & -0.001(2) & 0.046(7) \\ \text { H102 } & 0.147(2) & 0.0978(12) & 0.073(2) & 0.055(8) \\ \text { H103 } & 0.084(2) & 0.0315(13) & 0.067(2) & 0.059(8) \\ \text { H111 } & -0.132(2) & 0.0819(12) & 0.124(3) & 0.057(7) \\ \text { H112 } & -0.093(2) & 0.0238(14) & 0.197(3) & 0.066(9) \\ \text { H113 } & -0.132(2) & 0.0808(12) & 0.276(3) & 0.055(7) \\ \text { H121 } & 0.1781(18) & 0.1925(12) & 0.592(2) & 0.047(7) \\ \text { H131 } & 0.337(2) & 0.1868(14) & 0.701(3) & 0.065(8) \\ \text { H132 } & 0.340(2) & 0.1158(15) & 0.673(3) & 0.067(9) \\ \text { H133 } & 0.356(2) & 0.1629(14) & 0.558(3) & 0.077(10) \\ \text { H141 } & 0.161(2) & 0.1751(15) & 0.805(3) & 0.078(10) \\ \text { H142 } & 0.072(3) & 0.1404(15) & 0.741(3) & 0.079(10) \\ \text { H143 } & 0.178(3) & 0.099(2) & 0.789(4) & 0.132(16) \\ & & & & \end{array}$

The Temperature Factor has the Form of $\operatorname{Exp}(-\mathrm{T})$ Where $\mathrm{T}=8 *(\mathrm{Pi} * 2) * \mathrm{U} *(\mathrm{Sin}(\mathrm{Theta}) /$ Lambda $) * * 2$ for Isotropic Atoms

Table S4 - (An) isotropic Thermal Parameters for Compound 1

\begin{tabular}{|c|c|c|c|c|c|c|}
\hline Atom & $\mathrm{U}(1,1)$ or & $\mathrm{U}(2,2)$ & $\mathrm{U}(3,3)$ & $\mathrm{U}(2,3)$ & $\mathrm{U}(1,3)$ & $\mathrm{U}(1,2)$ \\
\hline--- & ------ & -- & -- & & & \\
\hline $\mathrm{Fe}$ & $0.0204(2)$ & $0.0190(2)$ & $0.0208(2)$ & 0 & $0.0043(2)$ & 0 \\
\hline Cl1 & $0.0314(4)$ & $0.0424(4)$ & $0.0228(3)$ & 0 & $0.0012(3)$ & 0 \\
\hline $\mathrm{Cl} 2$ & $0.0221(3)$ & $0.0420(4)$ & $0.0385(4)$ & 0 & $-0.0002(3)$ & 0 \\
\hline $\mathrm{N}$ & $0.0212(7)$ & $0.0166(7)$ & $0.0154(7)$ & $-0.0001(6)$ & $-0.0007(6)$ & $-0.0008(6)$ \\
\hline $\mathrm{C} 1$ & $0.0207(9)$ & $0.0217(9)$ & $0.0150(8)$ & $0.0009(7)$ & $-0.0016(7)$ & $-0.0020(7)$ \\
\hline $\mathrm{C} 2$ & $0.0291(11)$ & $0.0249(10)$ & $0.0428(12)$ & $0.0067(9)$ & $0.0115(10)$ & $-0.0011(8)$ \\
\hline $\mathrm{C} 3$ & $0.0212(8)$ & $0.0172(8)$ & $0.0234(9)$ & $0.0031(7)$ & $0.0046(7)$ & $0.0003(7)$ \\
\hline $\mathrm{C} 4$ & $0.0235(9)$ & $0.0200(9)$ & $0.0275(9)$ & $-0.0004(7)$ & $0.0027(7)$ & $-0.0012(7)$ \\
\hline $\mathrm{C} 5$ & $0.0341(11)$ & $0.0194(9)$ & $0.0398(11)$ & $-0.0043(9)$ & $0.0013(9)$ & $-0.0017(8)$ \\
\hline $\mathrm{C} 6$ & $0.0359(11)$ & $0.0178(9)$ & $0.0450(12)$ & $0.0073(9)$ & $0.0035(9)$ & $0.0024(8)$ \\
\hline $\mathrm{C} 7$ & $0.0326(10)$ & $0.0279(10)$ & $0.0316(11)$ & $0.0097(8)$ & $-0.0013(9)$ & $0.0033(9)$ \\
\hline $\mathrm{C} 8$ & $0.0296(10)$ & $0.0231(9)$ & $0.0246(9)$ & $0.0032(8)$ & $0.0012(8)$ & $0.0017(7)$ \\
\hline $\mathrm{C} 9$ & $0.0338(11)$ & $0.0215(10)$ & $0.0281(10)$ & $-0.0039(8)$ & $-0.0029(8)$ & $-0.0012(8)$ \\
\hline $\mathrm{C} 10$ & $0.0549(15)$ & $0.0455(14)$ & $0.0303(12)$ & $-0.0054(10)$ & $0.0016(11)$ & $0.0047(12)$ \\
\hline C11 & $0.0399(13)$ & $0.0497(15)$ & $0.0416(13)$ & $0.0035(11)$ & $-0.0126(11)$ & $-0.0105(11)$ \\
\hline C12 & $0.0588(14)$ & $0.0283(11)$ & $0.0284(11)$ & $-0.0003(9)$ & $-0.0166(10)$ & $0.0106(10)$ \\
\hline C13 & $0.0617(17)$ & $0.0488(16)$ & $0.0461(15)$ & $-0.0020(13)$ & $-0.0185(14)$ & $-0.0118(13)$ \\
\hline C14 & $0.067(2)$ & $0.108(3)$ & $0.0372(15)$ & $-0.0286(17)$ & $0.0007(14)$ & $0.007(2)$ \\
\hline
\end{tabular}


Anisotropic Atoms. Astar(i) are Reciprocal Axial Lengths and h(i) are the Reflection Indices. 
Table S5 - Bond Distances ( $\AA$ ) for Compound 1

$\begin{array}{llllll}\mathrm{Fe} & -\mathrm{C} 11 & 2.2280(7) & \mathrm{C} 2 & -\mathrm{H} 21 & 0.90(3) \\ \mathrm{Fe} & -\mathrm{C} 12 & 2.2163(7) & \mathrm{C} 2 & -\mathrm{H} 22 & 0.95(3) \\ \mathrm{Fe} & -\mathrm{N} & 2.1016(14) & \mathrm{C} 2 & -\mathrm{H} 23 & 0.95(2) \\ \mathrm{N} & -\mathrm{C} 1 & 1.281(2) & \mathrm{C} 5 & -\mathrm{H} 51 & 0.91(2) \\ \mathrm{N} & -\mathrm{C} 3 & 1.447(2) & \mathrm{C} 6 & -\mathrm{H} 61 & 0.96(2) \\ \mathrm{C} 1 & -\mathrm{C} 2 & 1.489(3) & \mathrm{C} 7 & -\mathrm{H} 71 & 0.94(2) \\ \mathrm{C} 1 & -\mathrm{C} 1-\mathrm{a} & 1.510(2) & \mathrm{C} 9 & -\mathrm{H} 91 & 0.94(2) \\ \mathrm{C} 3 & -\mathrm{C} 4 & 1.399(2) & \mathrm{C} 10 & -\mathrm{H} 101 & 0.99(2) \\ \mathrm{C} 3 & -\mathrm{C} 8 & 1.404(3) & \mathrm{C} 10 & -\mathrm{H} 102 & 0.97(3) \\ \mathrm{C} 4 & -\mathrm{C} 5 & 1.399(3) & \mathrm{C} 10 & -\mathrm{H} 103 & 1.02(3) \\ \mathrm{C} 4 & -\mathrm{C} 9 & 1.516(3) & \mathrm{C} 11 & -\mathrm{H} 111 & 0.95(3) \\ \mathrm{C} 5 & -\mathrm{C} 6 & 1.377(3) & \mathrm{C} 11 & -\mathrm{H} 112 & 0.96(3) \\ \mathrm{C} 6 & -\mathrm{C} 7 & 1.377(3) & \mathrm{C} 11 & -\mathrm{H} 113 & 0.98(3) \\ \mathrm{C} 7 & -\mathrm{C} 8 & 1.393(3) & \mathrm{C} 12 & -\mathrm{H} 121 & 0.92(2) \\ \mathrm{C} 8 & -\mathrm{C} 12 & 1.523(3) & \mathrm{C} 13 & -\mathrm{H} 131 & 0.97(3) \\ \mathrm{C} 9 & -\mathrm{C} 10 & 1.524(3) & \mathrm{C} 13 & -\mathrm{H} 132 & 0.93(3) \\ \mathrm{C} 9 & -\mathrm{C} 11 & 1.527(3) & \mathrm{C} 13 & -\mathrm{H} 133 & 0.99(3) \\ \mathrm{C} 12 & -\mathrm{C} 13 & 1.520(3) & \mathrm{C} 14 & -\mathrm{H} 141 & 0.98(3) \\ \mathrm{C} 12 & -\mathrm{C} 14 & 1.534(4) & \mathrm{C} 14 & -\mathrm{H} 142 & 0.90(4) \\ & & & \mathrm{C} 14 & -\mathrm{H} 143 & 1.08(4)\end{array}$

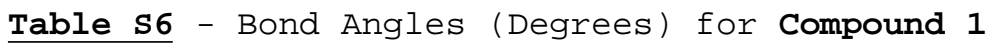

\begin{tabular}{|c|c|c|c|c|c|c|c|}
\hline $\mathrm{Cl1}$ & $-\mathrm{Fe}$ & $-\mathrm{Cl} 2$ & $115.82(3)$ & $\mathrm{C} 1$ & $-\mathrm{C} 2$ & $-\mathrm{H} 21$ & $110.4(14)$ \\
\hline Cl1 & $-\mathrm{Fe}$ & $-\mathrm{N}$ & $113.37(4)$ & $\mathrm{C} 1$ & $-\mathrm{C} 2$ & $-\mathrm{H} 22$ & $108.8(16)$ \\
\hline Cl1 & $-\mathrm{Fe}$ & $-N_{-} a$ & $113.37(4)$ & $\mathrm{C} 1$ & $-\mathrm{C} 2$ & $-\mathrm{H} 23$ & $110.9(14)$ \\
\hline Cl2 & $-\mathrm{Fe}$ & $-\mathrm{N}$ & $115.94(4)$ & H21 & $-\mathrm{C} 2$ & $-\mathrm{H} 22$ & $109(2)$ \\
\hline Cl2 & $-F e$ & $-\mathrm{N}_{-} \mathrm{a}$ & $115.94(4)$ & H21 & $-\mathrm{C} 2$ & $-\mathrm{H} 23$ & $110(2)$ \\
\hline $\mathrm{N}$ & $-\mathrm{Fe}$ & $-\mathrm{N}_{-} \mathrm{a}$ & $76.50(5)$ & $\mathrm{H} 22$ & $-\mathrm{C} 2$ & $-\mathrm{H} 23$ & $107(2)$ \\
\hline $\mathrm{Fe}$ & $-\mathrm{N}$ & $-\mathrm{C} 1$ & $114.07(11)$ & $\mathrm{C} 4$ & $-\mathrm{C} 5$ & $-\mathrm{H} 51$ & $119.0(14)$ \\
\hline $\mathrm{Fe}$ & $-\mathrm{N}$ & $-\mathrm{C} 3$ & $125.24(11)$ & $\mathrm{C} 6$ & $-\mathrm{C} 5$ & $-\mathrm{H} 51$ & $119.5(14)$ \\
\hline $\mathrm{C} 1$ & $-\mathrm{N}$ & $-\mathrm{C} 3$ & $120.63(14)$ & $\mathrm{C} 5$ & $-\mathrm{C} 6$ & $-\mathrm{H} 61$ & $120.7(13)$ \\
\hline $\mathrm{N}$ & $-\mathrm{C} 1$ & $-\mathrm{C} 2$ & $125.33(16)$ & $\mathrm{C} 7$ & $-\mathrm{C} 6$ & $-\mathrm{H} 61$ & $119.0(13)$ \\
\hline $\mathrm{N}$ & $-\mathrm{C} 1$ & $-\mathrm{C} 1 \_\mathrm{a}$ & $115.23(14)$ & $\mathrm{C} 6$ & $-C 7$ & $-\mathrm{H} 71$ & $119.8(13)$ \\
\hline $\mathrm{C} 1$ _a & $-\mathrm{C} 1$ & $-\mathrm{C} 2$ & $119.14(15)$ & $\mathrm{C} 8$ & $-\mathrm{C} 7$ & $-\mathrm{H} 71$ & $119.0(13)$ \\
\hline $\mathrm{N}$ & $-\mathrm{C} 3$ & $-\mathrm{C} 4$ & $120.20(15)$ & $\mathrm{C} 4$ & $-\mathrm{C} 9$ & $-\mathrm{H} 91$ & $107.5(11)$ \\
\hline $\mathrm{N}$ & $-\mathrm{C} 3$ & $-\mathrm{C} 8$ & $117.20(15)$ & $\mathrm{C} 10$ & $-\mathrm{C} 9$ & $-\mathrm{H} 91$ & $108.7(11)$ \\
\hline C4 & $-\mathrm{C} 3$ & $-\mathrm{C} 8$ & $122.54(16)$ & $\mathrm{C} 11$ & $-\mathrm{C} 9$ & $-\mathrm{H} 91$ & $107.1(12)$ \\
\hline C3 & $-\mathrm{C} 4$ & $-\mathrm{C} 5$ & $117.12(17)$ & C9 & $-\mathrm{C} 10$ & $-\mathrm{H} 101$ & $108.9(13)$ \\
\hline C3 & $-\mathrm{C} 4$ & $-\mathrm{C} 9$ & $122.64(16)$ & $\mathrm{C9}$ & $-\mathrm{C} 10$ & $-\mathrm{H} 102$ & $110.3(13)$ \\
\hline C5 & $-\mathrm{C} 4$ & $-\mathrm{C} 9$ & $120.23(16)$ & $\mathrm{C9}$ & $-\mathrm{C} 10$ & $-\mathrm{H} 103$ & $111.1(13)$ \\
\hline $\mathrm{C} 4$ & $-\mathrm{C} 5$ & $-\mathrm{C} 6$ & $121.41(18)$ & $\mathrm{H} 101$ & $-\mathrm{C} 10$ & $-\mathrm{H} 102$ & $109.5(19)$ \\
\hline $\mathrm{C} 5$ & $-\mathrm{C} 6$ & $-\mathrm{C} 7$ & $120.27(18)$ & $\mathrm{H} 101$ & $-\mathrm{C} 10$ & $-\mathrm{H} 103$ & $107.5(18)$ \\
\hline $\mathrm{C} 6$ & $-\mathrm{C} 7$ & $-\mathrm{C} 8$ & $121.16(19)$ & $\mathrm{H} 102$ & $-\mathrm{C} 10$ & $-\mathrm{H} 103$ & $109(2)$ \\
\hline $\mathrm{C} 3$ & $-\mathrm{C} 8$ & $-\mathrm{C} 7$ & $117.49(17)$ & C9 & $-\mathrm{C} 11$ & $-\mathrm{H} 111$ & $110.0(16)$ \\
\hline $\mathrm{C} 3$ & $-\mathrm{C} 8$ & $-\mathrm{C} 12$ & $122.75(17)$ & $\mathrm{C9}$ & $-\mathrm{C} 11$ & $-\mathrm{H} 112$ & $113.3(15)$ \\
\hline $\mathrm{C} 7$ & $-\mathrm{C} 8$ & $-\mathrm{C} 12$ & $119.76(17)$ & $\mathrm{C9}$ & $-\mathrm{C} 11$ & $-\mathrm{H} 113$ & $111.4(15)$ \\
\hline $\mathrm{C} 4$ & $-\mathrm{C} 9$ & $-\mathrm{C} 10$ & $111.70(17)$ & H111 & $-\mathrm{C} 11$ & $-\mathrm{H} 112$ & $107(2)$ \\
\hline $\mathrm{C} 4$ & $-\mathrm{C} 9$ & $-\mathrm{C} 11$ & $110.76(16)$ & H111 & $-\mathrm{C} 11$ & $-\mathrm{H} 113$ & $110(2)$ \\
\hline C10 & $-\mathrm{C} 9$ & $-\mathrm{C} 11$ & $110.96(17)$ & $\mathrm{H} 112$ & $-\mathrm{C} 11$ & $-\mathrm{H} 113$ & $106(2)$ \\
\hline \multirow[t]{9}{*}{$\mathrm{C} 8$} & $-\mathrm{C} 12$ & $-\mathrm{C} 13$ & $112.04(19)$ & $\mathrm{C} 8$ & $-\mathrm{C} 12$ & $-\mathrm{H} 121$ & $107.3(14)$ \\
\hline & & & & $\mathrm{C} 8$ & $-\mathrm{C} 12$ & $-\mathrm{C} 14$ & $110.6(2)$ \\
\hline & & & & $\mathrm{C} 13$ & $-\mathrm{C} 12$ & $-\mathrm{H} 121$ & $108.2(14)$ \\
\hline & & & & $\mathrm{C} 14$ & $-\mathrm{C} 12$ & $-\mathrm{H} 121$ & $108.2(14)$ \\
\hline & & & & $\mathrm{C} 12$ & $-\mathrm{C} 13$ & $-\mathrm{H} 131$ & $109.5(15)$ \\
\hline & & & & $\mathrm{C} 12$ & $-\mathrm{C} 13$ & $-\mathrm{H} 132$ & $109.1(16)$ \\
\hline & & & & C12 & $-\mathrm{C} 13$ & $-\mathrm{H} 133$ & $111.5(16)$ \\
\hline & & & & H131 & $-\mathrm{C} 13$ & $-\mathrm{H} 132$ & $108(3)$ \\
\hline & & & & $\mathrm{H} 131$ & $-\mathrm{C} 13$ & $-\mathrm{H} 133$ & $109(2)$ \\
\hline
\end{tabular}




$\begin{array}{llll}\text { H132 } & -\mathrm{C} 13 & -\mathrm{H} 133 & 110(2) \\ \mathrm{C} 12 & -\mathrm{C} 14 & -\mathrm{H} 141 & 106.3(17) \\ \mathrm{C} 12 & -\mathrm{C} 14 & -\mathrm{H} 142 & 113(2) \\ \mathrm{C} 12 & -\mathrm{C} 14 & -\mathrm{H} 143 & 108(2) \\ \mathrm{H} 141 & -\mathrm{C} 14 & -\mathrm{H} 142 & 106(3) \\ \mathrm{H} 141 & -\mathrm{C} 14 & -\mathrm{H} 143 & 107(3) \\ \mathrm{H} 142 & -\mathrm{C} 14 & -\mathrm{H} 143 & 117(3)\end{array}$


Table s7 - Predicted ${ }^{13} \mathrm{C}$ and ${ }^{1} \mathrm{H}$ NMR shifts (ppm) in the iron complex 1 from a dimer calculation.

\begin{tabular}{|c|ccc|}
\hline & $\delta_{\text {calc }}^{\text {dia }}(\mathbf{1})$ & $\delta_{\text {calc }}^{\text {dia }}(\mathbf{1})+\delta_{\text {calc }}^{\text {fc }}(\mathbf{1})$ & $\delta^{\text {obs }}(\mathbf{2})+\delta_{\text {calc }}^{\text {fc }}(\mathbf{1})$ \\
\hline C1 & 167 & 117 & 122.5 \\
C2 & -6 & 297 & 323.8 \\
C3 & 144 & 139 & 132.8 \\
C4 & 140 & 202 & 201.4 \\
C5 & 115 & 13 & 22.5 \\
C6 & 117 & 238 & 247.2 \\
C7 & 116 & -52 & -44.3 \\
C8 & 141 & 120 & 118.4 \\
C9 & 13 & -67 & -51.4 \\
C10 & -1 & 87 & 113.4 \\
C11 & -5 & 64 & 92.3 \\
C12 & 12 & -42 & -26.6 \\
C13 & -5 & 106 & 135.5 \\
C14 & 0 & 28 & 52.9 \\
\hline H2 & -4 & 3 & 9.6 \\
H5 & 3 & 20 & 23.5 \\
H6 & 5 & -23 & -21.5 \\
H7 & 4 & 8 & 10.7 \\
H9 & -2 & -2 & 2.7 \\
H10 & -3 & -1 & 3.3 \\
H11 & -5 & -7 & -1.6 \\
H12 & -3 & 4 & 10.1 \\
H13 & -4 & -2 & 3.5 \\
H14 & -3 & -1 & 3.3 \\
\hline
\end{tabular}



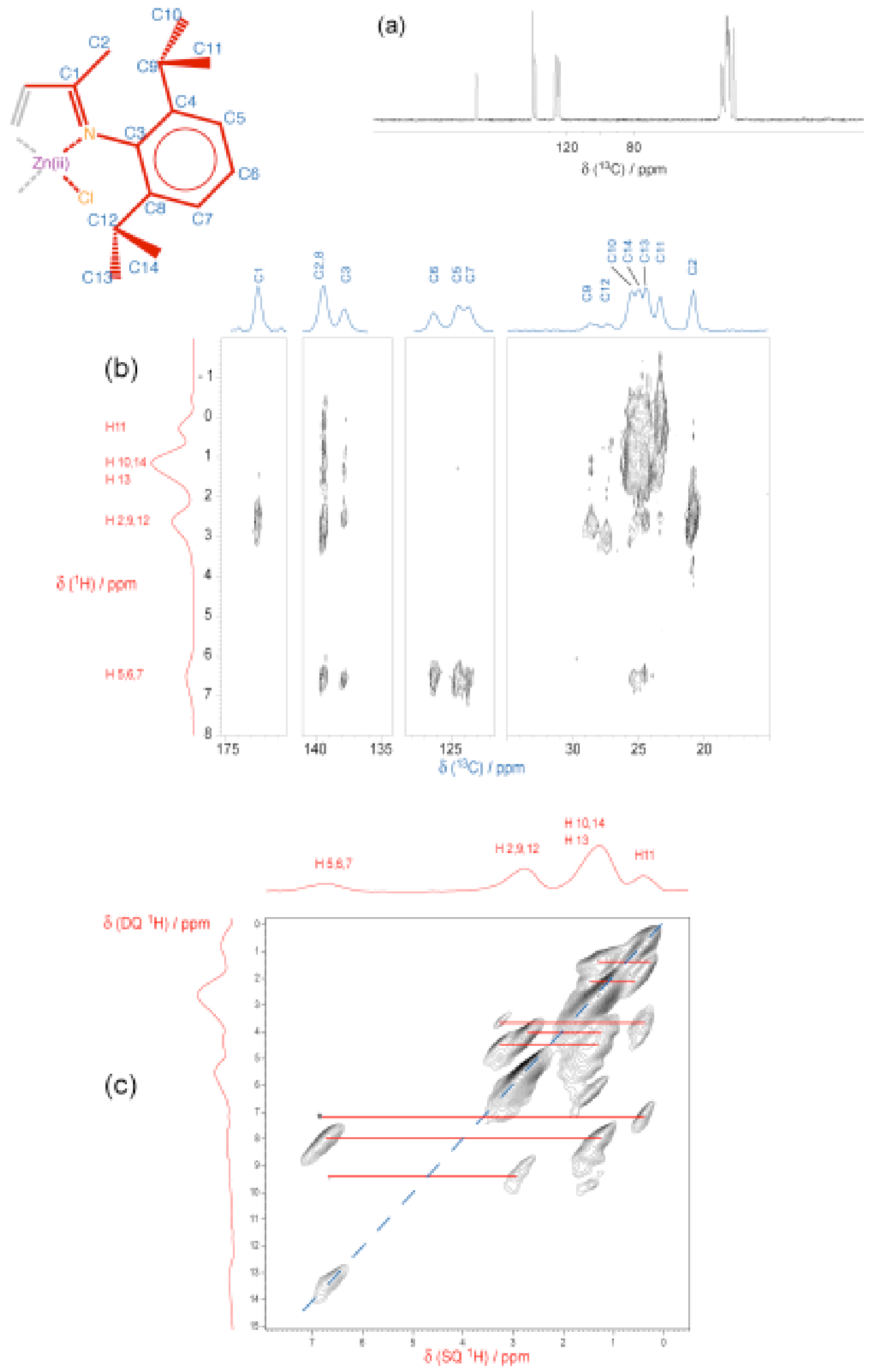

Figure S1 - a) ${ }^{13} \mathrm{C}$ MAS NMR spectra $(500 \mathrm{MHz})$ of 2; (b) ${ }^{1} \mathrm{H}-{ }^{13} \mathrm{C}$ dipolar HETCOR and (c) ${ }^{1} \mathrm{H}$ DQ CRAMPS correlations of 2 . 
All the spectra were acquired on compound 2 at a temperature of 30 deg under 20 $\mathrm{kHz}$ MAS. For a ${ }^{1} \mathrm{H}^{13} \mathrm{C} \mathrm{CP}$, a tangential amplitude modulated $\mathrm{RF}$ field(1) centered at $100 \mathrm{kHz}$ was applied on ${ }^{1} \mathrm{H}$, while the carbon field on $13 \mathrm{C}$ was matched to obtain optimal signal; an optimal contact time was $1 \mathrm{~ms}$. A decoupling field of $100 \mathrm{kHz}$ was applied to protons according to the TPPM scheme(2) during acquisition. In a ${ }^{1} \mathrm{H}-$ ${ }^{13} \mathrm{C}$ dipolar HETCOR experiment, $100 \mathrm{kHz}$ windowless DUMBO-1 22 homonuclear dipolar decoupling(3) was applied during $1 \mathrm{H}$ evolution in $t_{1}$. A scaling factor of 0.58 during $t_{1}$ was experimentally determined applying the same sequence on a standard ${ }^{13} \mathrm{C}^{-}$ uniformly labeled glycine sample. A total of $256 t_{1}$ increments were collected $\left(t_{1 \max }=32.7 \mathrm{~ms} ; t_{2 \max }=45 \mathrm{~ms}\right)$, with 128 scans each and a recycle delay of $2.5 \mathrm{~s}$ (total recording time $23 \mathrm{~h}$ ). Quadrature detection during $\mathrm{f}_{1}$ was achieved using the TPPI method. (4) An exponential line broadening of $10 \mathrm{~Hz}$ was applied in the two dimensions before Fourier transformation.

For a ${ }^{1} \mathrm{H}$ DQ CRAMPS experiment, ${ }^{(5)}$ double quantum excitation and reconversion were performed through two POST-C7(6) blocks of 42.8 us (corresponding to 3 basic POST-C7 elements each). Homonuclear ${ }^{1} \mathrm{H}$ decoupling $(100 \mathrm{kHz})$ was achieved by the DUMBO-1 $1_{22}$ scheme ${ }^{(3)}$ during $f_{1}$ as described for experiment (a), while a windowed w-DUMBO-1 homonuclear decoupling scheme ${ }^{(7)}$ was applied during $t_{2}$ acquisition. The scaling factors for DUMBO-1 during $f_{1}$ and $f_{2}$ were determined experimentally as 0.58 and 0.84 , respectively. A total of $80 t_{1}$ increments were collected $\left(t_{1 \max }=8.4 \mathrm{~ms} ; t_{2 \max }=\right.$ $13.7 \mathrm{~ms}$ ), with 48 scans each and a recycle delay of $2 \mathrm{~s}$ (total recording time 2 h). Quadrature detection during $f_{1}$ was achieved using the states-TPPI method. An exponential line broadening of $10 \mathrm{~Hz}$ was applied in the two dimensions before Fourier transformation.

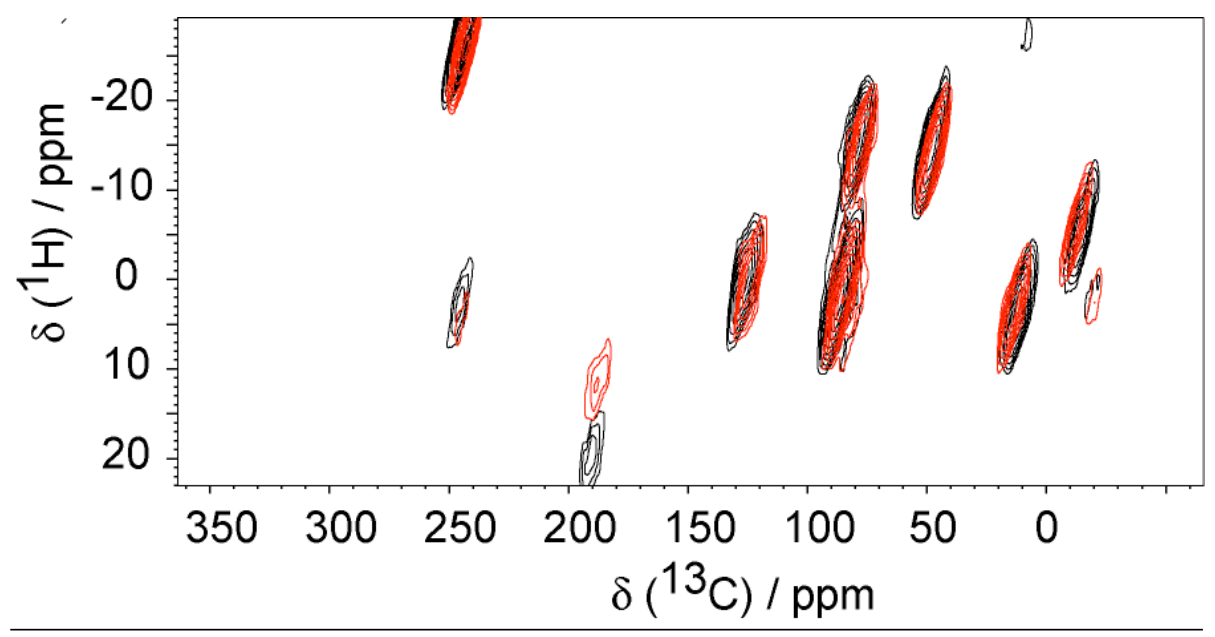

Figure S2 - Comparison between two rotor-synchronized 1H-13C TEDoR correlations of 1, acquired under $33 \mathrm{kHz}$ (black contours) and $30 \mathrm{kHz}$ (red contours) MAS $(\mathrm{T}=303 \mathrm{~K} ; \mathrm{B} 0=11.7 \mathrm{~T})$. The comparison allows the correct evaluation of the shift of the C2-H2 group, which is aliased, and moves $3 \mathrm{kHz}$ upfield upon changing the MAS rate.

The assignment of the $\mathrm{C}_{2} \mathrm{H}_{2}$ signal was also confirmed by recording the same experiment on a sample selectively ${ }^{2} \mathrm{H}$-labeled on the $\alpha$-imine methyl (kindly provided by P.J Chirik). 


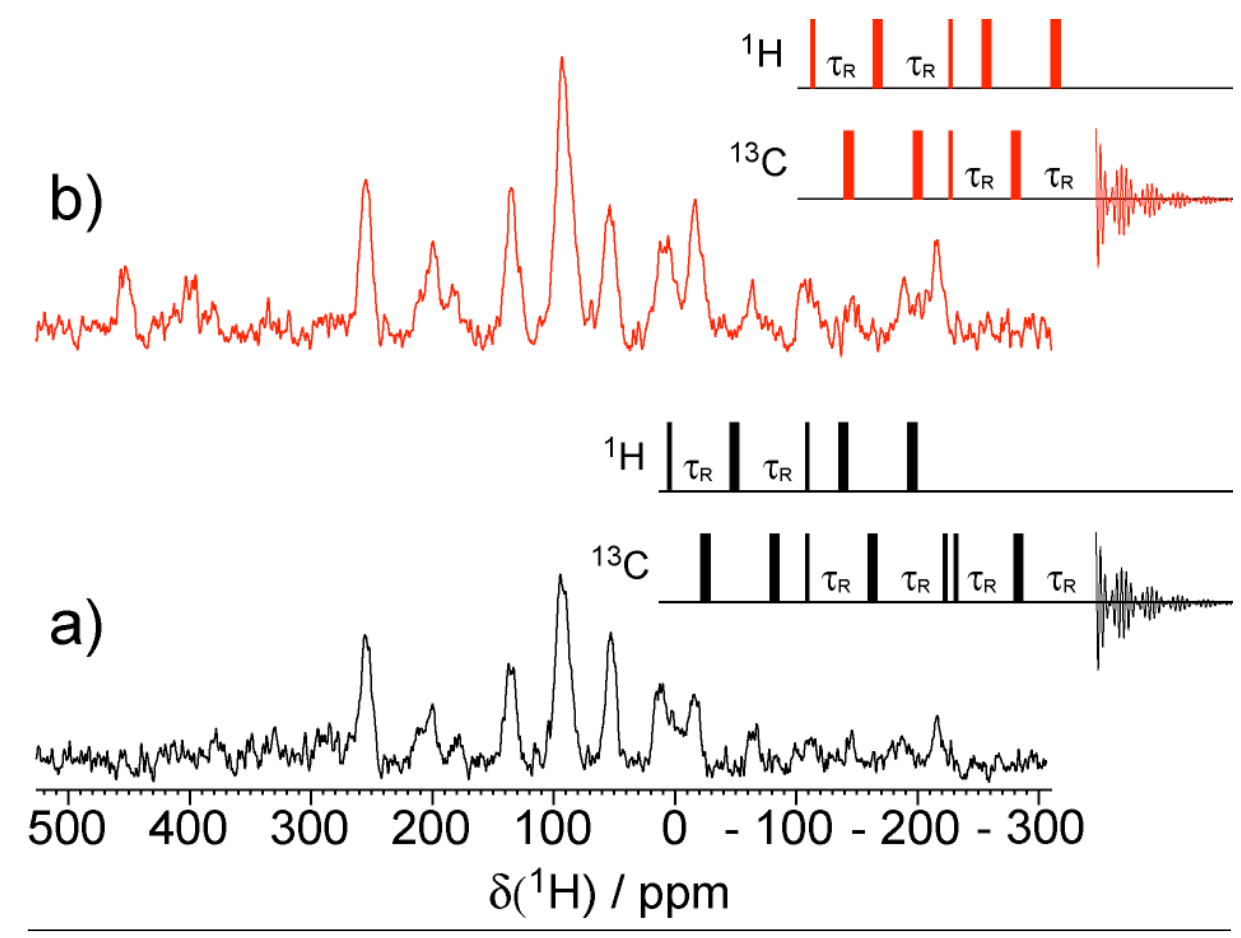

S11

Figure S3 - Comparison between a standard 1H-13C TEDOR correlation of 1 (a) and a modified version, lacking the z-filter and spin-echo elements before $13 \mathrm{C}$ acquisition (b). ). Narrow and wide rectangles denote $90^{\circ}$ and $180^{\circ}$ pulses, respectively; _R represents the rotor period. (33 kHz MAS; $\mathrm{T}=303 \mathrm{~K} ; \mathrm{B} 0=11.7 \mathrm{~T})$.

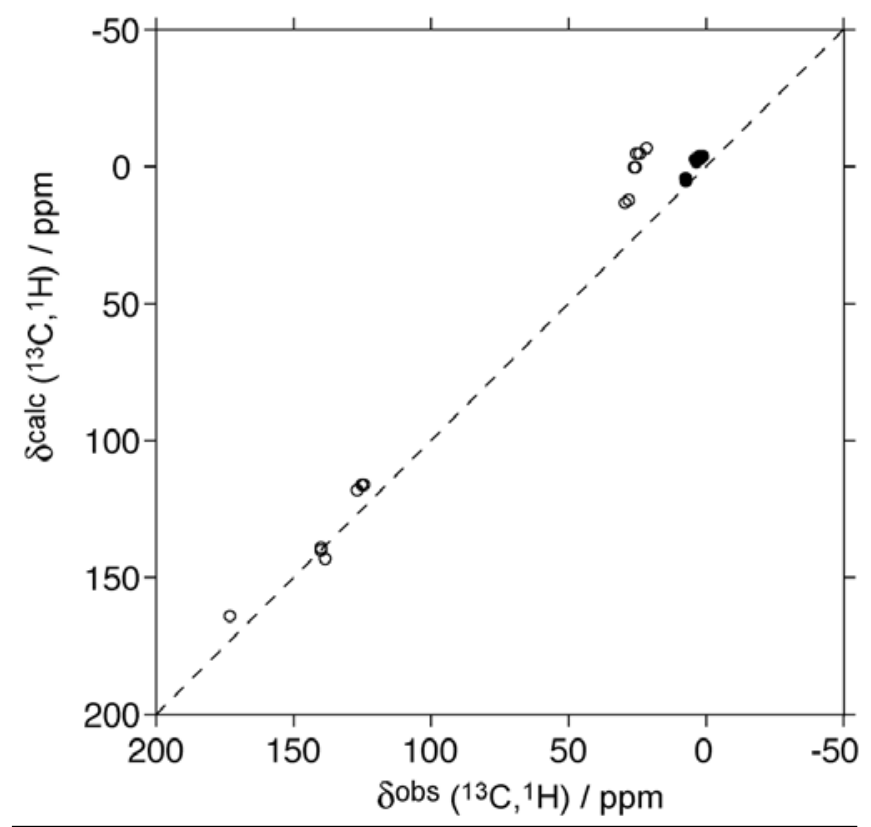

Figure S4 - Correlation betweeen experimental shifts for complex 2 and calculated diamagnetic shifts for complex 1 .

${ }^{13} \mathrm{C}$ are represented by open symbols and ${ }^{1} \mathrm{H}$ by filled symbols. 
(a)
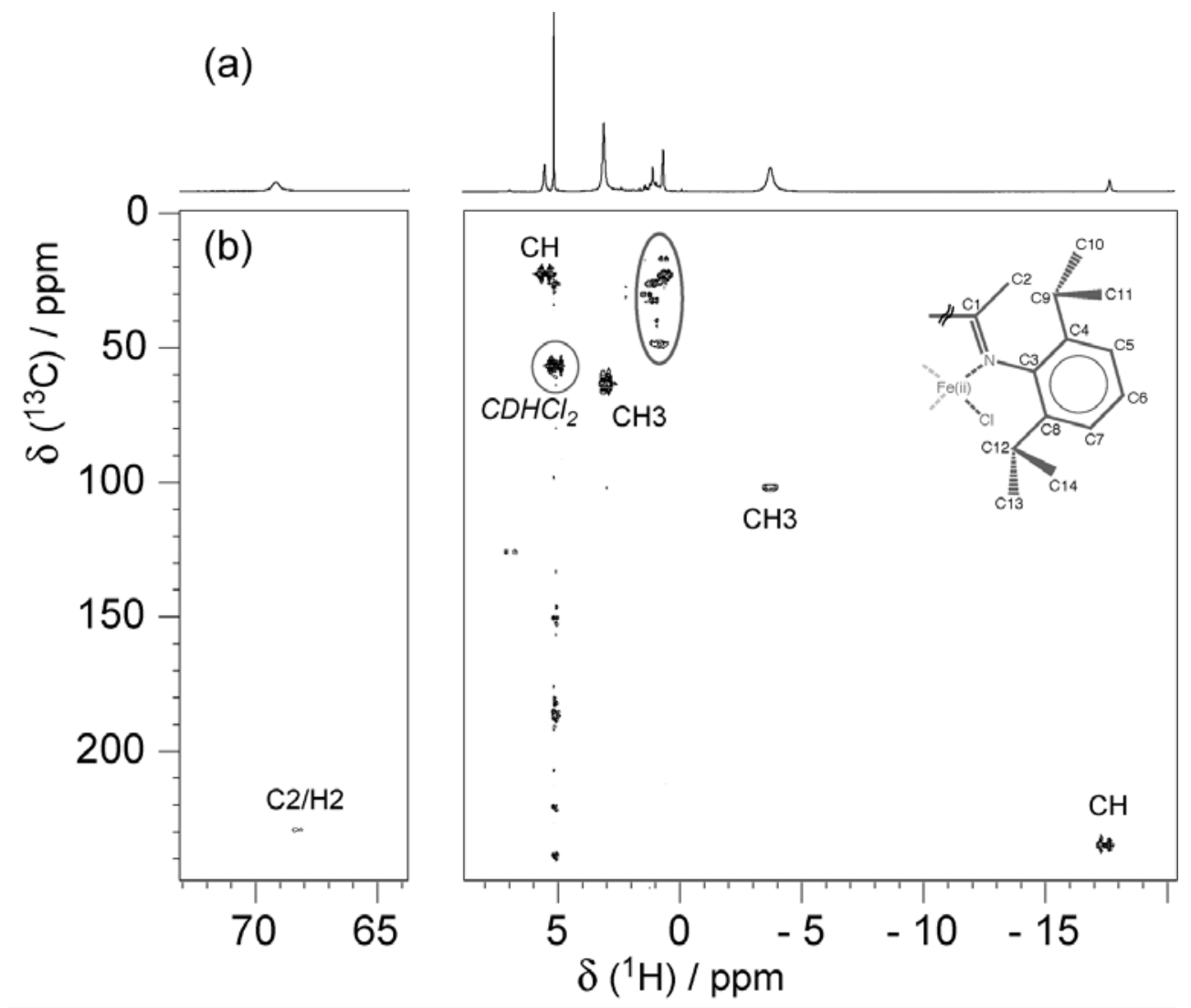

Figure S5 - Liquid-state NMR spectra of 1 dissolved in $\mathrm{CH}_{2} \mathrm{Cl}_{2}$. (a) ${ }^{1} \mathrm{H}$ NMR; (b) ${ }^{1} \mathrm{H},{ }^{13} \mathrm{C}-\mathrm{HMQC}$. The spectra were recorded at 30 deg on a Varian Inova 500 NMR spectrometer using the following parameters: $t_{1 \max }=50 \mathrm{~ms} ; t_{2 \max }=$ $150 \mathrm{~ms}, 120 \mathrm{ppm}$ sweep width in the ${ }^{1} \mathrm{H}$ dimension, total recording time $64 \mathrm{~h}$. Quadrature detection during $f_{1}$ was achieved using the states method. Transverse relaxation during the pulse sequence due to paramagnetism was minimized by reducing the MQC excitation delay to $1.1 \mathrm{~ms}$. Resonances are labeled according to the ${ }^{1} \mathrm{H}$ assignment reported by Bart et al. (8). Two circles identify the residual solvent signals, as well as cross-peaks from the uncomplexed, diamagnetic ligand.

\section{Bibliography}

(1) Hediger, S.; Meier, B. H.; Kurur, N. D.; Bodenhausen, G. and Ernst, R. R., Nmr Cross-Polarization by Adiabatic Passage through the Hartmann-Hahn Condition (Aphh). Chem. Phys. Lett. 1994, 223, 283-288.

(2) Bennett, A. E.; Rienstra, C. M.; Auger, M.; Lakshmi, K. V. and Griffin, R. G., Heteronuclear Decoupling in Rotating Solids. J. Chem. Phys. 1995, 103, 69516958.

(3) De Paepe, G.; Elena, B. and Emsley, L., Characterization of heteronuclear decoupling through proton spin dynamics in solid-state nuclear magnetic resonance spectroscopy. J. Chem. Phys. 2004, 121, 3165-80.

(4) Marion, D. and Wüthrich, K., Application of phase sensitive two-dimensional correlated spectroscopy (COSY) for measurements of $1 \mathrm{H}-1 \mathrm{H}$ spin-spin coupling constants in proteins. Biochem Biophys Res Commun 1983, 113, 967-74.

(5) Brown, S. P.; Lesage, A.; Elena, B. and Emsley, L., Probing proton-proton proximities in the solid state: high-resolution two-dimensional $1 \mathrm{H}-1 \mathrm{H}$ doublequantum CRAMPS NMR spectroscopy. J. Am. Chem. Soc. 2004, 126, 13230-1. 
(6) Hohwy, M.; Jakobsen, H. J.; Eden, M.; Levitt, M. H. and Nielsen, N. C., Broadband dipolar recoupling in the nuclear magnetic resonance of rotating solids: A compensated C7 pulse sequence. J. Chem. Phys. 1998, 108, 2686-2694.

(7) Lesage, A.; Sakellariou, D.; Hediger, S.; Elena, B.; Charmont, P.; Steuernagel, S. and Emsley, L., Experimental aspects of proton NMR spectroscopy in solids using phase-modulated homonuclear dipolar decoupling. J Magn Reson 2003, $163,105-13$.

(8) Bart, S. C.; Hawrelak, E. J.; Schmisseur, A. K.; Lobkovsky, E.; Chirik, P. J., Synthesis, reactivity and solid-state structures of four-coordinate iron(II) and manganese(II) alkyl complexes. Organometallics 2004, 23, 237.

\section{Complete ref. 44 from the Main Text}

(44) Gaussian 03, Revision C.02; Frisch, M. J.; Trucks, G. W.; Schlegel, H. B.; Scuseria, G. E.; Robb, M. A.; Cheeseman, J. R.; Montgomery, J., J. A.; Vreven, T.; Kudin, K. N.; Burant, J. C.; Millam, J. M.; Iyengar, S. S.; Tomasi, J.; Barone, V.; Mennucci, B.; Cossi, M.; Scalmani, G.; Rega, N.; Petersson, G. A.; Nakatsuji, H.; Hada, M.; Ehara, M.; Toyota, K.; Fukuda, R.; Hasegawa, J.; Ishida, M.; Nakajima, T.; Honda, Y.; Kitao, O.; Nakai, H.; Klene, M.; Li, X.; Knox, J. E.; Hratchian, H. P.; Cross, J. B.; Adamo, C.; Jaramillo, J.; Gomperts, R.; Stratmann, R. E.; Yazyev, O.; Austin, A. J.; Cammi, R.; Pomelli, C.; Ochterski, J. W.; Ayala, P. Y.; Morokuma, K.; Voth, G. A.; Salvador, P.; Dannenberg, J. J.; Zakrzewski, V. G.; Dapprich, S.; Daniels, A. D.; Strain, M. C.; Farkas, O.; Malick, D. K.; Rabuck, A. D.; Raghavachari, K.; Foresman, J. B.; Ortiz, J. V.; Cui, Q.; Baboul, A. G.; Clifford, S.; Cioslowski, J.; Stefanov, B. B.; Liu, G.; Liashenko, A.; Piskorz, P.; Komaromi, I.; Martin, R. L.; Fox, D. J.; Keith, T.; Al-Laham, M. A.; Peng, C. Y.; Nanayakkara, A.; Challacombe, M.; Gill, P. M. W.; Johnson, B.; Chen, W.; Wong, M. W.; Gonzalez, C. and Pople, J. A.; Gaussian, Inc., Wallingford CT, 2004. 